\title{
A Two-stage Vector Perturbation Scheme for Adaptive Modulation in Downlink MU-MIMO
}

\author{
Ang Li, Student Member, IEEE, and Christos Masouros, Senior Member, IEEE
}

\begin{abstract}
Conventional vector perturbation (VP) is not directly applicable to adaptive modulation while other existing algorithms are suboptimal due to the reduced search dimension of perturbation vectors. In this paper, by applying a simple transformation to the conventional VP operation, the search dimension for the proposed joint vector perturbation is made equal to that of conventional VP, and therefore the performance advantages of VP still hold in this scenario. Furthermore, to reduce the computational complexity, a joint constructive VP scheme is introduced by exploiting constructive interference to simplify the VP operation. By doing so, the sophisticated search for perturbation vectors is partially replaced by a quadratic programming problem, therefore saving significant computational complexity. Our analysis and results show that the proposed scheme offers an improved performance-complexity tradeoff compared to conventional VP approaches by means of the measurement in energy efficiency.
\end{abstract}

Index Terms-MIMO, vector perturbation, precoding, adaptive modulation, two-stage perturbation

\section{INTRODUCTION}

$\mathbf{I}$ $\mathrm{N}$ recent multiple-input-multiple-output (MIMO) communication systems, precoding techniques have been widely studied and simple forms of precoding have appeared in communication standards [1][2]. Existing precoding approaches range from linear precoding [3] to non-linear precoding [4]. Non-linear vector perturbation (VP) based precoding in [4][7] improves the performance of linear channel inversion precoding schemes. However, one drawback of conventional VP scheme is that it does not apply to scenarios where multiple modulation types are employed by different users due to the fact that the modulo base $\tau=2|\mathrm{C}|_{\max }+\Delta$ is modulation dependent, where $|\mathrm{C}|_{\max }$ is the absolute value of the constellation symbol with the maximum magnitude and $\Delta$ denotes the minimum Euclidean distance between constellation points. In [8], VP is combined with block-diagonalized (BD) precoding as BD-VP to enable VP technique applicable for adaptive modulation scenario. Nevertheless, due to the operation of singular value decomposition (SVD), BD-VP is computationally inefficient. To circumvent this, a low-complexity BD-VP

Copyright (c) 2015 IEEE. Personal use of this material is permitted. However, permission to use this material for any other purposes must be obtained from the IEEE by sending a request to pubs-permissions@ieee.org.

Manuscript received March 16, 2015; revised June 16, and August 20, 2015; accepted October 6, 2015.This work was supported by the Royal Academy of Engineering, UK and the Engineering and Physical Sciences Research Council (EPSRC) project EP/M014150/1.

A. Li and C. Masouros are with the Dept. of Electronic and Electrical Eng., University College London, Torrington Place, London, WC1E 7JE, UK (e-mail: ang.li.14@ucl.ac.uk, chris.masouros@ieee.org). scheme is introduced in [9] and a user-grouping VP (UG$\mathrm{VP}$ ) is also proposed to improve the performance of BDVP. However, both BD-VP and UG-VP are suboptimal to conventional VP due to the reduced search dimensions.

In [10], a joint vector perturbation algorithm is proposed that enables VP applicable to adaptive modulation scenarios without degrading the performance. By scaling the constellation of different modulation types and the corresponding channel matrix, the modulo base $\tau$ is made common for all modulation types, and therefore a full dimension search for the perturbation vectors could be performed. However, the computational complexity of the proposed scheme in [10] is comparable to conventional VP schemes. Therefore, to reduce the complexity, in this paper a two-stage joint constructive vector perturbation scheme is further introduced. In the first stage, the search for the perturbation vectors is exclusively limited for users applying QAM modulation while in the second stage, the constructive VP [11] is performed for users applying PSK modulation where the search space is limited to the areas that are constructive to the transmit symbols. These are the areas where the perturbation vectors can increase the distances to the decision thresholds of the constellation and therefore can increase the detection performance. Moreover, as the constructive perturbation vectors need not be removed because they will benefit the symbol detection, the perturbation vectors need not be integers. Therefore, instead of using the sophisticated sphere encoding technique, a quadratic programming method could be applied, thus saving much computational complexity especially when the search dimension is large.

For reasons of clarity we summarize the contributions of the paper as:

1) We propose a reduced complexity two-stage VP scheme for adaptive modulation scenarios.

2) We derive the performance of the proposed scheme analytically in the presence of imperfect CSI.

3) We characterize the performance-complexity tradeoff for the proposed and conventional schemes by introducing an energy efficiency metric.

Notation: $E(\cdot),(\cdot)^{T},(\cdot)^{H},(\cdot)^{-1}$ and $(\cdot)^{\dagger}$ denote expectation, transpose, conjugate transpose, inverse and MoorePenrose inverse respectively. $\|\cdot\|$ denotes the Frobenius Norm, $I_{n}$ is the $n \times n$ identity matrix and $\mathbf{0}$ denotes zero matrix or vector. $\mathbf{R}^{n \times n}$ represents a $n \times n$ matrix in the real set and $\mathbf{C Z}^{n}$ represents $n \times 1$ vectors in the complex integer set. 


\section{SySTEM MODEL AND CONVENTIONAL VP}

\section{A. MU-MIMO Channel Model}

A multiuser multi-stream MIMO downlink system is considered where the base station communicates with $K$ users simultaneously. It is assumed that the base station is equipped with $N_{t}$ antennas with each user $k$ equipped with $n_{k} \geq 1$ antennas. The total number of receive antennas is therefore defined as $N_{\mathrm{r}}=\sum_{k=1}^{K} n_{k}$ with $N_{r} \leq N_{t}$. A flat fading channel is assumed and modeled by the $n_{k} \times N_{t}$ channel matrix $\mathbf{H}_{k}$ for user $k$, and the channel matrix from the base station to all users can be expressed as $\mathbf{H}=\left[\mathbf{H}_{1}^{T}, \mathbf{H}_{2}^{T}, \ldots, \mathbf{H}_{K}^{T}\right]^{T}$. Then, the received signal at $k$ th user is

$$
\mathbf{y}_{k}=\mathbf{H}_{k} \mathbf{x}_{k}+\mathbf{H}_{k} \sum_{i=1, i \neq k}^{K} \mathbf{x}_{i}+\mathbf{w}_{k}
$$

where $\mathbf{x}_{k}$ is the precoded signal to transmit and $\mathbf{w}_{k}$ is the additive complex Gaussian noise vector with zero mean and unit variance.

\section{B. Vector Perturbation Precoding}

VP Precoding employs a channel inversion to form the precoding matrix and then applies a perturbation on the transmitted symbols. For each user $k$, the transmitted signal is then given as

$$
\mathbf{x}_{k}=\sqrt{\frac{P}{\beta}} \mathbf{F}_{k}\left(\mathbf{s}_{k}+\tau \mathbf{l}_{k}^{*}\right)
$$

where $\mathbf{F}_{k}$ is the precoding matrix of $k$ th user and $\mathbf{s}_{k}$ denotes the data symbols of $k$ th user. $\beta=\|\mathbf{F} \cdot(\mathbf{s}+\tau \mathbf{l})\|^{2}$ denotes the transmit power scaling factor so that $E\left(\|\mathbf{x}\|^{2}\right)=P$ where $\mathbf{s}=$ $\left[\mathbf{s}_{1}^{T}, \mathbf{s}_{2}^{T}, \ldots, \mathbf{s}_{K}^{T}\right]^{T}$ and $\mathbf{x}=\left[\mathbf{x}_{1}^{T}, \mathbf{x}_{2}^{T}, \ldots, \mathbf{x}_{K}^{T}\right]^{T} . \mathbf{l}^{*} \in \mathbf{C Z}^{N_{t}}$ is the perturbation vector. Usually in VP, the precoding matrix could be in the form of channel inversion and channel inversion can be viewed as a special case where $\mathbf{l}^{*}=\mathbf{0}$. The received signal of VP for user $k$ after the channel can be calculated as

$$
\mathbf{y}_{k}=\sqrt{\frac{P}{\beta}}\left(\mathbf{s}_{k}+\tau \mathbf{l}_{k}^{*}\right)+\mathbf{w}_{k}
$$

At the receiver, the signal is first scaled back to eliminate the effect of the transmit scaling factor, and then fed to a modulo operator to remove the perturbation vector [4]. Ignoring the effect of modulo loss, the output symbols of modulo operation can be expressed as

$$
\begin{aligned}
\mathbf{r}_{k} & =\bmod _{\tau}\left[\sqrt{\frac{\beta}{P}} \mathbf{y}_{k}\right]=\bmod _{\tau}\left[\mathbf{s}_{k}+\tau \mathbf{l}_{k}^{*}+\sqrt{\frac{\beta}{P}} \mathbf{w}_{k}\right] \\
& =\mathbf{s}_{k}+\mathbf{n}_{k}
\end{aligned}
$$

where

$$
\bmod _{\tau}[x]=f_{\tau}(\Re(x))+j \cdot f_{\tau}(\Im(x))
$$

and

$$
f_{\tau}(x)=x-\left\lfloor\frac{x+\tau / 2}{\tau}\right\rfloor \cdot \tau
$$

$\mathbf{n}_{k}$ denotes the noise for user $k$ after the modulo operation.

\section{Proposed Two-Stage Joint Constructive VECTOR PERTURBATION (JCVP)}

\section{A. Joint Vector Perturbation (JVP)}

In conventional VP algorithm, the perturbation vector $\mathbf{l}^{\text {opt }}$ is searched over $N_{r}$ dimensions to minimize the total transmit power. However, due to the fact that different modulation has a different value of $\tau$, conventional VP cannot be directly applied to scenarios where users employ multiple modulation types. In order to keep the performance advantage of conventional VP, we employ a joint perturbation technique that retains the search dimensions as $N_{r}$. Without loss of generality, we assume there are multiple modulation types applied, denoted as $\mathcal{A}, \mathcal{B}, \mathcal{C}, \ldots, \mathcal{M}$. For modulation type $i$, the total number of symbols applying this kind of modulation is denoted as $N_{i}$ and $\sum_{i=\mathcal{A}}^{\mathcal{M}} N_{i}=N_{r}$. For conventional VP, $\mathbf{l}^{\text {opt }}=\underset{\mathbf{l} \in \mathbf{C} \mathbf{Z}^{N_{r}}}{\arg \min }\|\mathbf{F}(\mathbf{s}+\tau \mathbf{l})\|^{2}$ where $\mathbf{F}$ is the precoding matrix. Rewrite $\mathbf{F}=\left[\mathbf{F}_{\mathcal{A}}, \mathbf{F}_{\mathcal{B}}, \ldots, \mathbf{F}_{\mathcal{M}}\right], \mathbf{s}+$ $\tau \mathbf{l}=\left[\left(\mathbf{s}_{\mathcal{A}}+\tau_{\mathcal{A}} \mathbf{l}_{\mathcal{A}}\right)^{T},\left(\mathbf{s}_{\mathcal{B}}+\tau_{\mathcal{B}} \mathbf{l}_{\mathcal{B}}\right)^{T}, \ldots,\left(\mathbf{s}_{\mathcal{M}}+\tau_{\mathcal{M}} \mathbf{l}_{\mathcal{M}}\right)^{T}\right]^{T}$, where $\mathbf{s}_{i}$ denotes the symbols from the constellation of modulation type $i$ and $\mathbf{F}_{i}$ is the corresponding precoding matrix. Then, we can reformulate the transmit signal norm as

$$
\begin{array}{r}
\|\mathbf{F}(\mathbf{s}+\tau \mathbf{l})\|^{2}=\left\|\left[\mathbf{F}_{\mathcal{A}}, \mathbf{F}_{\mathcal{B}}, \ldots, \mathbf{F}_{\mathcal{M}}\right] \cdot(\mathbf{s}+\tau \mathbf{l})\right\|^{2} \\
=\left\|\sum_{i=\mathcal{A}}^{\mathcal{M}} \frac{\tau_{i}}{\tau_{0}} \cdot \frac{\tau_{0}}{\tau_{i}} \mathbf{F}_{i}\left(\mathbf{s}_{i}+\tau_{i} \mathbf{l}_{i}\right)\right\|^{2}=\left\|\sum_{i=\mathcal{A}}^{\mathcal{M}} \frac{\tau_{i}}{\tau_{0}} \mathbf{F}_{i}\left(\frac{\tau_{0}}{\tau_{i}} \mathbf{s}_{i}+\tau_{0} \mathbf{l}_{i}\right)\right\|^{2}
\end{array}
$$

where $\mathbf{l}_{i}$ is the perturbation vector for modulation type $i$ and $\tau_{0}$ denotes the modulo base to be used. Based on the derivation above, it is worth noting that there is no special restriction for the value of $\tau_{0}$ as when the symbols are scaled, the corresponding channel is also scaled accordingly. By defining $\mathbf{G}=\left[\frac{\tau_{\mathcal{A}}}{\tau_{0}} \mathbf{F}_{\mathcal{A}}, \ldots, \frac{\tau_{\mathcal{M}}}{\tau_{0}} \mathbf{F}_{\mathcal{M}}\right]$ and $\mathbf{t}=\left[\frac{\tau_{0}}{\tau_{\mathcal{A}}} s_{\mathcal{A}}^{T}, \ldots, \frac{\tau_{0}}{\tau_{\mathcal{M}}} s_{\mathcal{M}}^{T}\right]^{T}$,

can be transformed into

$$
\|\mathbf{F}(\mathbf{s}+\tau \mathbf{l})\|^{2}=\left\|\mathbf{G}\left(\mathbf{t}+\tau_{0} \mathbf{l}\right)\right\|^{2}
$$

With this transformation, the search for the perturbation vectors in adaptive modulation scenario is kept the same as conventional VP scheme. Intuitively, this algorithm functions as follows: by scaling the constellation of the modulation and the corresponding channel matrix, different modulation types have the same value of $\tau$ without any change in the value of the signal norm so that the vector perturbation could be performed jointly.

\section{B. Complexity Reduction by Joint Constructive Vector Pertur- bation (JCVP)}

Although JVP preserves the performance benefits of conventional VP, it also preserves the high computational complexity of conventional VP. In order to reduce the complexity, 
the constructive VP (CVP) first introduced in [11] is applied in the adaptive modulation scenario. By limiting the search space for the perturbation vectors to the constructive areas where the symbols have increased distances to the decision thresholds of the constellation, the perturbation vectors for CVP at the receivers need not be removed, and therefore need not be integers. As conventional CVP is not applicable to our scheme, we propose a modification in the following. For notational simplicity, let us first decompose the channel and information symbols into real and imaginary parts as

$$
\hat{\mathbf{H}}=\left[\begin{array}{cc}
\Re(\mathbf{H}) & -\Im(\mathbf{H}) \\
\Im(\mathbf{H}) & \Re(\mathbf{H})
\end{array}\right]
$$

and

$$
\hat{\mathbf{s}}=\left[\begin{array}{ll}
\Re(\mathbf{s})^{T} & \Im \\
(\mathbf{s})^{T}
\end{array}\right]^{T}
$$

Then, the transmit signal is modified by applying a diagonal scaling matrix $\boldsymbol{\Theta} \in \mathbf{R}^{2 N_{t} \times 2 N_{t}}$ and can be formulated as

$$
\hat{\mathbf{x}}=\sqrt{\frac{P}{\beta}} \mathbf{F}(\boldsymbol{\Theta} \hat{\mathbf{s}})
$$

In this case, the scaling factor is obtained as

$$
\beta=\|\mathbf{F}(\boldsymbol{\Theta} \hat{\mathbf{s}})\|^{2}
$$

It is well established in the literature that the computational complexity of conventional VP grows exponentially with the increase in search dimension, while it is shown that the complexity of CVP reduces to the polynomial complexity [11]. In order to preserve the complexity advantage of CVP in the adaptive modulation scenario, a two-stage joint constructive vector perturbation (JCVP) scheme is then introduced. By using the concept of selective perturbation, only the symbols from QAM constellations are perturbed in the first stage, followed by a partial CVP scheme for the symbols from PSK constellations. To be specific, in the first stage, the optimization for the perturbation vector of (8) is modified as

$$
\mathbf{l}^{*}=\arg \min \left\|\overline{\mathbf{G}}\left(\overline{\mathbf{t}}+\tau_{0}\left[\begin{array}{c}
\mathbf{0}_{N_{t}-L} \\
\mathbf{l}_{L}
\end{array}\right]\right)\right\|^{2}
$$

where $L$ denotes the total number of symbols from QAM constellations, $\overline{\mathbf{t}}$ and $\overline{\mathbf{G}}$ denote the appropriate reordering of the transmit symbols and the corresponding matrix $\mathbf{G}$ after performing JVP. Rewrite $\overline{\mathbf{G}}=\left[\overline{\mathbf{G}}_{N_{t}-L}, \overline{\mathbf{G}}_{L}\right]$, (13) can be further expressed as

$$
\mathbf{l}^{*}=\arg \min \left\|\overline{\mathbf{G}} \overline{\mathbf{t}}+\tau_{0} \overline{\mathbf{G}}_{L} \mathbf{l}_{L}\right\|^{2}
$$

Then, (14) is a $L$-dimensional VP problem and can be solved by standard sphere search techniques.

In the second stage, a partial CVP is applied for symbols from PSK modulations to minimize the scaling factor. As mentioned before, we exploit the illustration of JVP and the constructive interference as shown in Fig. 1, where we assume $\tau_{0}$ is chosen as $\tau_{16 Q A M}$ for simplicity. According to the definition of $\tau$, the length of the fold lines $\tau_{16 Q A M}=$ $d_{1}^{16 Q A M}+d_{2}^{16 Q A M}$ and $\tau_{Q P S K}=d_{1}^{Q P S K}+d_{2}^{Q P S K}$ denotes the value of the modulo bases for 16QAM and QPSK, respectively. We scale the constellation of QPSK by $\tau_{16 Q A M} / \tau_{Q P S K}$ such that $\frac{\tau_{16 Q A M}}{\tau_{s Q P S K}}=\frac{d_{1}^{16 Q A M}+d_{2}^{16 Q A M}}{d^{s Q P S K}+d_{2}^{s Q P S K}}=1$, and therefore JVP could be performed. Moreover, the blue zone denotes the initial constructive area of QPSK while the green zone denotes the constructive area of SQPSK, as defined in [11]. Denote $\overline{\mathbf{u}}=\overline{\mathbf{t}}+\tau_{0}\left[\begin{array}{c}\mathbf{0}_{N_{t}-L} \\ \mathbf{l}_{L}^{*}\end{array}\right]=\left[\mathbf{u}_{N_{t}-L}^{T}, \mathbf{u}_{L}^{T}\right]^{T}$ and $\mathbf{u}_{N_{t}-L}^{T}$ are the symbols to be optimized in this stage. Then, following a similar step of CVP, a diagonal scaling matrix $\overline{\mathbf{\Theta}} \in \mathbf{R}^{2 N_{t} \times 2 N_{t}}$ is applied. Noticing that the scaling is only applied for symbols from PSK constellations, the scaling factor can be formulated as

$$
\beta=\|\hat{\overline{\mathbf{G}}}(\overline{\boldsymbol{\Theta}} \hat{\overline{\mathbf{u}}})\|^{2}
$$

where $\hat{\overline{\mathbf{G}}}$ and $\hat{\overline{\mathbf{u}}}$ are obtained by expanding the real and imaginary part of $\overline{\mathbf{G}}$ and $\overline{\mathbf{u}}$. Then, by defining $\bar{\theta}=\operatorname{diag}(\overline{\mathbf{\Theta}})$, $\hat{\overline{\mathbf{U}}}=\operatorname{diag}(\hat{\overline{\mathbf{u}}}), \mathbf{R}^{1 / 2}=\hat{\overline{\mathbf{G}}} \hat{\overline{\mathbf{U}}}$ and with some rearrangements, a simplified quadratic programming can be obtained as

$$
\begin{array}{cc} 
& \bar{\theta}=\arg \min _{\bar{\theta}} \bar{\theta}^{T} \mathbf{R} \bar{\theta} \\
\text { s.t. } & \bar{\theta}_{1: N_{t}-L} \geq 1, \bar{\theta}_{N_{t}+1: 2 N_{t}-L} \geq 1 \\
& \bar{\theta}_{N_{t}-L+1: N_{t}}=1, \bar{\theta}_{2 N_{t}-L+1: 2 N_{t}}=1
\end{array}
$$

where $\bar{\theta}_{i}$ is the $i$ th diagonal element of $\bar{\Theta}$. The second line of the constraints ensures that QAM symbols are not scaled. As we already split the transmit symbols into the real part and imaginary part, we must ensure the scaling factor of QAM symbols for both is equal to 1 . In the second stage, the symbols from QAM constellations are not scaled, and this is guaranteed by setting the corresponding $\bar{\theta}$ equal to 1 , as seen from (16). Finally, the transmit scaling factor is obtained as

$$
\begin{aligned}
\beta & =\|\overline{\mathbf{G}} \hat{\boldsymbol{\Theta}} \overline{\mathbf{u}}\|^{2}=\left\|\left[\overline{\mathbf{G}}_{N_{t}-L}, \overline{\mathbf{G}}_{L}\right] \cdot\left[\begin{array}{c}
\hat{\mathbf{\Theta}}_{N_{t}-L} \cdot \overline{\mathbf{t}}_{N_{t}-L} \\
\overline{\mathbf{t}}_{L}+\tau_{0} \mathbf{l}_{L}^{*}
\end{array}\right]\right\|^{2} \\
& =\left\|\overline{\mathbf{G}}_{N_{t}-L} \hat{\boldsymbol{\Theta}}_{N_{t}-L} \overline{\mathbf{t}}_{N_{t}-L}+\overline{\mathbf{G}}_{L} \cdot\left(\overline{\mathbf{t}}_{L}+\tau_{0} \mathbf{l}_{L}^{*}\right)\right\|^{2}
\end{aligned}
$$

where $\hat{\boldsymbol{\Theta}}$ is defined as the complex equivalent of $\overline{\boldsymbol{\Theta}}$.

Remark: It is natural to consider that these two steps can be performed conversely. However, it will be shown in the simulation results that applying conventional VP followed by CVP always results in a better performance for JCVP. This can be explained as follows. In the second stage, compared with conventional VP that only searches limited integers, CVP searches the entire constructive areas such that there is a higher possibility that the scaling factor is minimized.

\section{Performance Analysis in the Presence of CSI ERRORS}

In this section we present a mathematical performance analysis for JCVP in the presence of CSI errors. For analytical tractability, we focus on the case where the precoding matrix $\mathbf{F}=\mathbf{H}^{\dagger}$. In realistic communication systems where there exist 


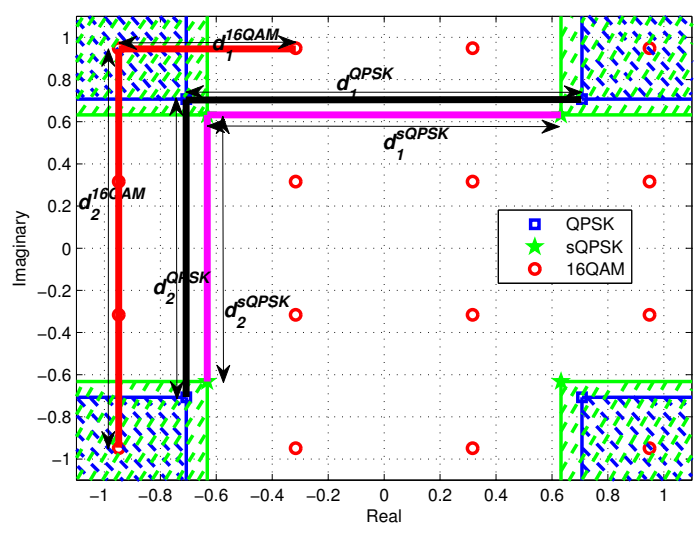

Fig. 1. Illustration of the constructive areas for QPSK and sQPSK in the normalized constellation

channel state information (CSI) errors, we assume the errors are inversely proportional to the transmit SNR and therefore can be modeled as

$$
\hat{\mathbf{H}}=\mathbf{H}+\mathbf{E}
$$

with $\mathbf{E} \sim \mathcal{C N}\left(0, \eta \cdot \mathbf{I}_{N_{t}}\right)$, statistically independent to $\mathbf{H}$. Therefore, the channel is modeled as [12]

$$
\mathbf{H}=\frac{1}{1+\eta} \cdot \hat{\mathbf{H}}+\mathbf{Q}
$$

with $\mathbf{Q} \sim \mathcal{C N}\left(0, \frac{\eta}{1+\eta} \cdot \mathbf{I}_{N_{t}}\right)$. As we assume the system operates in TDD mode and the CSI is directly measured at the transmitter using reciprocity, $\eta$ is defined as $\eta=\alpha \cdot\left(\frac{P}{\sigma^{2}}\right)^{-1}$ with $\alpha$ being the channel error coefficient and $\frac{P}{\sigma^{2}}$ being the transmit SNR [11]. In this paper, we apply the constructive perturbation for PSK users while retaining the conventional perturbation operation for QAM users. Therefore, at the receiver, the received SNR for PSK users and QAM users must be calculated independently. Firstly, the received signals of users applying PSK modulation can be expressed as

$$
\begin{aligned}
\mathbf{y}_{P S K} & =\sqrt{\frac{P}{\beta}}\left(\frac{1}{1+\eta} \hat{\mathbf{H}}+\mathbf{Q}\right) \hat{\mathbf{H}}^{\dagger} \hat{\mathbf{\Theta}} \mathbf{s}_{P S K}+\mathbf{w} \\
& =\frac{1}{1+\eta} \sqrt{\frac{P}{\beta}} \hat{\mathbf{\Theta}} \mathbf{s}_{P S K}+\sqrt{\frac{P}{\beta}} \mathbf{Q} \hat{\mathbf{H}}^{\dagger} \hat{\mathbf{\Theta}} \mathbf{s}_{P S K}+\mathbf{w}
\end{aligned}
$$

where $\mathbf{w}$ denotes the noise vector that consists of the noise term for each user. We then define

$$
\hat{\mathbf{w}}_{P S K}=\sqrt{\frac{P}{\beta}} \mathbf{Q} \hat{\mathbf{H}}^{\dagger} \hat{\boldsymbol{\Theta}} \mathbf{s}_{P S K}+\mathbf{w}
$$

as the equivalent noise term and $\hat{\mathbf{w}}_{P S K} \sim \mathcal{C N}\left(0, v_{P S K}\right)$ where $v_{P S K}$ is given as [12]

$$
v_{P S K}=\frac{P}{\beta} \cdot \frac{\eta}{1+\eta} \cdot \beta \cdot \bar{\theta}_{i}^{2}+\sigma^{2}=\frac{\eta P \bar{\theta}_{i}^{2}}{1+\eta}+\sigma^{2}
$$

Then, the SNR of users applying PSK modulation can be calculated as

$$
\gamma_{i}=\frac{1}{(1+\eta)^{2} \beta} \cdot \frac{P \bar{\theta}_{i}^{2}}{\left(\frac{\eta P \bar{\theta}_{i}^{2}}{1+\eta}+\sigma^{2}\right)}
$$

where term $(1+\eta)^{2} \beta$ is due to the fact that the signals must be scaled back before demodulation.

Then, for users applying QAM modulation, by expanding (4), scaling back the signals by $(1+\eta) \sqrt{\frac{\beta}{P}}$ and considering the modulo operation the received signal can be expressed as

$$
\begin{aligned}
\mathbf{y}_{Q A M} & =\mathbf{s}_{Q A M}+\tau \mathbf{l}+(1+\eta) \mathbf{Q} \hat{\mathbf{H}}^{\dagger}\left(\mathbf{s}_{Q A M}+\tau \mathbf{l}\right) \\
& +(1+\eta) \sqrt{\frac{\beta}{P}} \mathbf{w}
\end{aligned}
$$

where we define

$$
\hat{\mathbf{w}}_{Q A M}=(1+\eta) \mathbf{Q} \hat{\mathbf{H}}^{\dagger}\left(\mathbf{s}_{Q A M}+\tau \mathbf{l}\right)+(1+\eta) \sqrt{\frac{\beta}{P}} \mathbf{w}
$$

as the equivalent noise term. Based on [12], the distribution of $\hat{\mathbf{w}}_{Q A M}$ is conditoned on $\hat{\mathbf{H}}^{\dagger}$ and $\hat{\mathbf{w}}_{Q A M} \sim \mathcal{C N}\left(0, v_{Q A M}\right)$ where $v_{Q A M}$ is given as [12]

$$
\begin{aligned}
v_{Q A M} & =(1+\eta)^{2} \frac{\eta}{1+\eta} \beta+(1+\eta)^{2} \frac{\beta}{P} \sigma^{2} \\
& =\beta\left[(1+\eta) \eta+(1+\eta)^{2} \frac{\sigma^{2}}{P}\right]
\end{aligned}
$$

Therefore the SNR for QAM symbols can be expressed by

$$
\gamma_{i}=\frac{P}{\beta\left[(1+\eta) \eta+(1+\eta)^{2} \frac{\sigma^{2}}{P}\right]}
$$

Then, the achievable sum rate is obtained as

$$
R=\sum_{i=1}^{K} \log _{2}\left(1+\gamma_{i}\right)
$$

\section{Complexity Analysis}

The complexity is measured in terms of the required number of floating point operation (flops) [13]-[14]. Inversing the $n_{k} \times n_{k}$ matrix and the $N_{r} \times N_{t}$ matrix requires $4 n_{k}^{3} / 3$ flops and $4 N_{t}^{3} / 3$ flops respectively by Gauss-Jordan elimination. The search for the optimal perturbation vectors within $n$ dimensions requires $O\left(n^{6}\right)$ flops where $O()$ defines the order of numerical operations [12], and a $M$ dimensional quadratic programming consumes $O\left((2 M)^{3} N\right)$ where $N$ is the length of the quadratic programming and can be expressed as [11]

$$
N=\log _{2}\left(\max \left|\psi_{i, j}\right|+1\right)+\log _{2}(4 M)+1
$$

where $\psi_{i, j}$ is the $i, j$-th element of matrix $\mathbf{R}$. With the proposed scheme in this paper, the computational complexity for PSK symbols and QAM symbols are different. Therefore, the complexity for different modulation types must be calculated separately. The total complexity is then obtained as the sum of 
the complexity of PSK symbols and QAM symbols, expressed as

$$
\begin{aligned}
C & =O\left(4 N_{t}^{3} / 3\right)+O\left(L^{6}\right)+O\left(8\left(N_{t}-L\right)^{3} \cdot N\right) \\
& =O\left(4 N_{t}^{3} / 3\right)+O\left(L^{6}\right)+O\left(8\left(N_{t}-L\right)^{3}\right. \\
& \left.\times\left(\log _{2}\left(\max \left|\psi_{i, j}\right|+1\right)+\log _{2}(4 M)+1\right)\right)
\end{aligned}
$$

\section{ENERGY EFFICIENCY}

To evaluate the usefulness of each VP algorithm, we explore the performance-complexity tradeoff by means of the resulting energy efficiency. In this section, the energy efficiency of proposed JVP is compared with previous VP algorithms in terms of the number of transmit antennas. We define the energy efficiency of the communication link as the bit rate per total transmit power consumed [7][10], shown as

$$
E E=\frac{R}{P_{P A}+N_{t} \cdot P_{0}+p_{c} \cdot C}
$$

where $P_{P A}=(\xi / \eta-1) P$ in Watts is the power consumed at the power amplifier to produce the total transmit power $P$ of the entire antenna elements and is therefore independent of the number of BS antennas. $\eta$ is the power amplifier efficiency and $\xi=3 \frac{\sqrt{M}-1}{\sqrt{M}+1}$ for $M$-QAM is the modulation dependent peak to average power ratio (PAPR). $P_{0}=P_{m i x}+P_{f i l t}+P_{D A C}$ denotes the power consumption of the mixers and filters and the digital-to-analog converter (DAC), which is regarded as constant in this paper. From [10], the values of each parameter are as follows: $\eta=0.35, P_{m i x}=30.3 \mathrm{~mW}, P_{f i l t}=2.5 \mathrm{~mW}$, and $P_{D A C}=1.6 \mathrm{~mW}$ which yields $P_{0}=34.4 \mathrm{~mW} . p_{c}$ in $\mathrm{mW} /$ Mflops denotes the power consumption per $10^{6}$ flops of the digital signal processor (DSP). In this paper we use $p_{c}=1 / 12.8 \mathrm{~mW} /$ Mflops [10].

\section{NUMERICAL RESULTS}

In this section we present Monte Carlo simulations of the proposed JCVP where we assume $P=1$, the number of transmit antennas is $N_{t}=8$. There are $K=4$ users and each is equipped with $n_{k}=2$ antennas. The channel error coefficient is assumed as $\alpha=1$. Except for the conventional VP where all users apply QPSK or 16QAM, it is assumed that user 1,2 apply QPSK and user 3,4 apply 16QAM. Above parameters and assumptions remain the same throughout the following simulations. It is intuitive that the benefits of JVP and JCVP extend to other adaptive modulation scenarios.

Fig. 2 shows the bit error rate (BER) performance of two JCVP schemes where CVP-VP denotes applying CVP first followed by VP and VP-CVP denotes applying VP first followed by CVP. From this figure, it is observed that BDVP and UG-VP are suboptimal compared with JVP and the proposed JCVP. Moreover, it can be seen that VP-CVP outperforms CVP-VP, and this is because the scaling factor of VPCVP is smaller than that of CVP-VP by searching the entire constructive areas rather than limited integers. Moreover, the performance of users applying PSK modulations is better than JVP due to the constructive interference, while the users

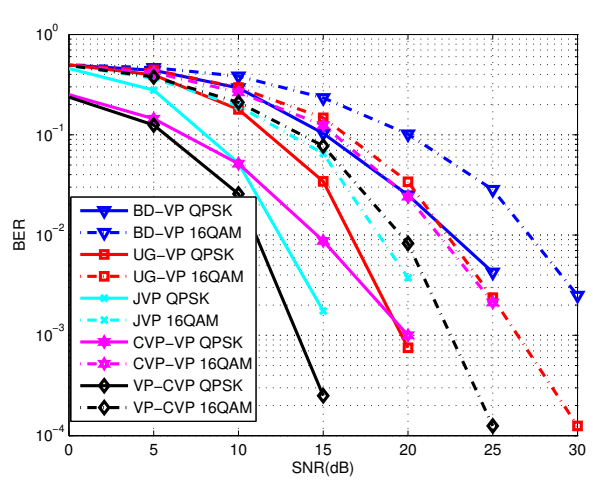

Fig. 2. BER performance of VP schemes, perfect CSI, $N_{t}=8, K=4$, $n_{k}=2$

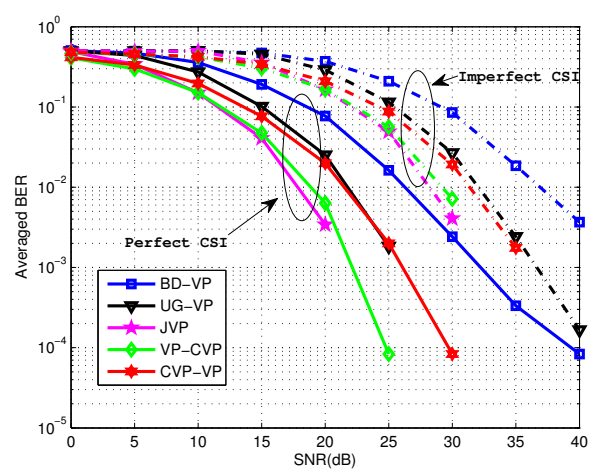

Fig. 3. Average BER performance of different VP schemes, $N_{t}=8, K=4$, $n_{k}=2, \alpha=1$

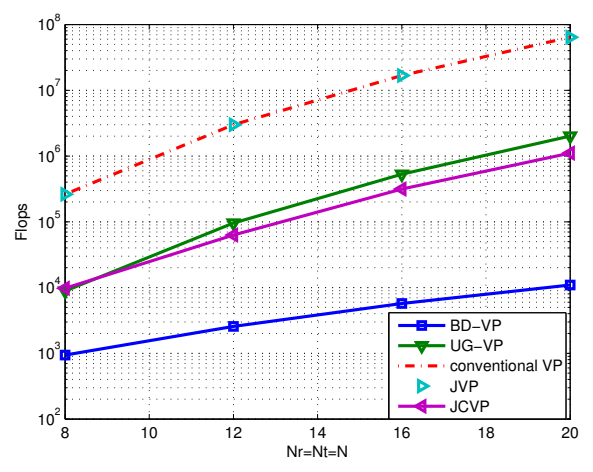

Fig. 4. Computational complexity of different VP schemes, $N_{t}=N_{r}$

applying QAM modulations suffer a slight performance loss compared with JVP due to the increased value of scaling factor.

Fig. 3 compares the BER performance averaged over all modulations of BD-VP, UG-VP, JVP, VP-CVP and CVP-VP with perfect CSI and imperfect CSI, respectively. From this figure, a similar BER trend could be seen and all VP schemes suffer a performance degradation due to the channel estimation error. It is also observed that VP-CVP outperforms CVP-VP in terms of average BER, however VP-CVP is worse than JVP because JCVP has a inferior performance for users applying QAM modulation. 


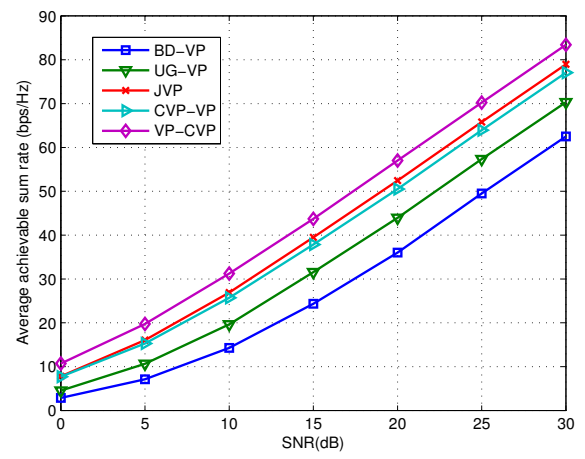

Fig. 5. Achievable sum rate of different VP schemes, $N_{t}=8, K=4$, $n_{k}=2$

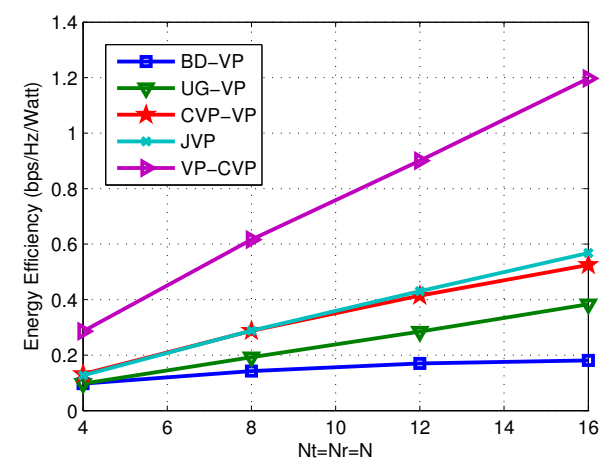

Fig. 6. Energy efficiency of different VP schemes, $N_{t}=N_{r}, P=43 \mathrm{dBm}$

Fig. 4 shows the computational complexity of different VP schemes where VP-CVP and CVP-VP are represented by JCVP as they consume the same complexity. It can be seen that compared with conventional VP and JVP that consumes a very high complexity, the proposed scheme costs much less complexity while provides a comparable BER performance, which is more significant with the increasing number of antennas. Therefore, it is shown that the proposed scheme could provide a more favorable performance-complexity tradeoff.

Fig. 5 compares the achievable sum rate for different VP schemes. As can be seen from the figure, the proposed VPCVP offers the largest sum rate, followed by JVP. It is worth noting that although the scaling factor of VP-CVP is larger than that of JVP, the constructive interference contributes to the sum rate, therefore leading to the superior sum rate performance of VP-CVP. The scaling factor of CVP-VP is large and the constructive interference could not compensate the noise amplification effect, which leads to a lower sum rate performance.

Fig. 6 shows the energy efficiency for different VP schemes. It is observed that BD-VP and UG-VP have a low energy efficiency performance because of the low sum rate. Moreover, it is seen that CVP-VP has a similar performance to JVP. Although the sum rate of CVP-VP is less than that of JVP as shown by Fig. 5, CVP-VP consumes less complexity, therefore leading to a similar energy efficiency performance. It is worth noting that VP-CVP achieves the highest energy efficiency, which is due to the superior performance of the sum rate as illustrated by Fig. 5 and a relatively low complexity in Fig. 4. Therefore, the superior energy efficiency performance of VP-CVP shows that the proposed technique offers a more favorable performance-complexity tradeoff.

\section{CONCLUSION}

In this paper, a joint constructive vector perturbation scheme for multiuser MIMO downlink system that enables the application of VP techniques to adaptive modulation scenarios is proposed. Furthermore, by limiting the search space to the constructive areas, the perturbation vectors for PSK modulations need not be removed and thus need not be integers. Therefore, a quadratic programming method could instead be applied for symbols from PSK modulations, saving significant computational complexity. Simulation results validate the proposed technique and show that the performance of users applying PSK modulations is much better than that of JVP and the performance of users applying QAM modulations is slightly worse than that of JVP, while the computational complexity is much less, leading to a more favorable performance-complexity tradeoff, validated by the energy efficiency performance.

\section{REFERENCES}

[1] Evolved Universal Terrestrial Radio Access (E-UTRA); Physical Channels and Modulation, 3GPP TS 36.211, V 8.2.0 (2008-03), Release 8.

[2] Evolved Universal Terrestrial Radio Access (E-UTRA):LTE Physical Layer; General Description, 3GPP TS 36.201, V11.1.0 (2008-03), Release 11.

[3] M. A. Khojastepour X. Wang, and M. Madihian, "Design of Multiuser Downlink Linear Precoding Systems With Quantized Feedback," IEEE Trans. Vehicular Technology, vol. 58, no. 9, pp. 4828-4836, Feb. 2009.

[4] A. Razi, D. J. Ryan, I. B. Collings, and J. Yuan, "Sum rates, rate allocation, and user scheduling for multi-user MIMO vector perturbation precoding," IEEE Trans. Wireless Cотmu., vol. 9, no. 1, pp. 356-365, Jan, 2010.

[5] C. Masouros, M. Sellathurai, and T. Ratnarajah, "Computationally Efficient Vector Perturbation Precoding Using Thresholded Optimization," IEEE Trans. Commun., vol. 61, no. 5, pp. 1880-1890, May 2013.

[6] C. Masouros, M. Sellathurai, and T. Ratnarajah, "A Low-Complexity Sequential Encoder for Threshold Vector Perturbation," IEEE Coтmunications Letters, vol. 17, no. 12, pp. 2225-2228, December 2013.

[7] C. Masouros, M. Sellathurai, and T. Ratnarajah, "Maximizing Energy Efficiency in the Vector Precoded MU-MISO Downlink by Selective Perturbation," IEEE Trans. Wireless Commun., vol. 13, no. 9, pp. 49744984, September 2014.

[8] C.-B. Chae, S. Shim, and R. W. Heath, "Block diagonalized vector perturbation for multiuse MIMO systems," IEEE Trans. Wireless Commun., vol. 7, no. 11, pp. 4051-4057, Nov 2008.

[9] R. Chen. C. Li, J. Li and Y. Zhang, "Low Complexity User Grouping Vector Perturbation," IEEE Wireless Communications Letters, vol. 1, no. 3, pp. 189-192, June 2012.

[10] A. Li, and C. Masouros, "A Constellation Scaling Approach to Vector Perturbation for Adaptive Modulation in MU-MIMO," IEEE Wireless Commun. Lett., vol. 4, no. 3, pp. 289-292, June 2015.

[11] C. Masouros, M. Sellathurai, and T. Ratnarajah, "Vector Perturbation Based on Symbol Scaling for Limited Feedback MISO Downlinks," IEEE. Trans. Signal Processing., vol. 62, no. 3, pp. 562-571, Feb 2014.

[12] J. Maurer, J. Jalden, D. Seethaler, and G. Matz, "Vector Perturbation Precoding Revisted," IEEE Trans. Signal Process., vol. 59, no. 1, pp. 315-328, October 2010.

[13] G. H. Golub and C. F. V. Loan, Matrix Computations, 3rd edition. The Johns Hopkins University Press, 1989.

[14] H. Sung, S. CR. Lee, and I. Lee, "Generalized channel inversion methods for multiuser MIMO systems," IEEE Trans. Commun., vol. 57, no. 11, pp. 3489-3499, Nov 2009. 